\title{
Testing for urinary tract infections in residential care
}

Urinary tract infections (UTIs) are a cause of considerable anxiety for patients, relatives of elderly people and health care workers alike. They complicate comorbidity and long term conditions, cause discomfort and confusion in older people, predispose them to falls and can lead to hospital admission (Alzheimer's Society, 2011; Beveridge et al 2011)

Frailty (a lack of physiological reserve and multiple degenerative changes in an elders body ) alongside $50 \%$ less total body water content in older people, (compared with younger adults) puts the former more at risk of dehydration and delirium (confusion). This and poor hydration from not drinking enough will put them in a higher risk group for UTIs the use of diuretics and other medication might also increase this risk (Lally and Crome 2007) again increasing dehydration and susceptibility to UTIs.

\section{Symptoms of a UTI}

The symptoms seen in UTI can vary enormously and in younger adults they can vary from mild discomfort to passing urine that is bloodstained and accompanied by back or loin pain and shivers (rigors). In older people there may be urine that is now offensive, new incontinence and often confusion (this is termed as 'delirium' by NICE 2015) or being 'off legs' (a sudden deterioration in mobility). Falls that cannot be attributed to other factors should prompt an investigation into fluid intake and urine output including assessment of vital signs and the use of a dipstick to test the urine.

If the results of these investigations are negative other causes should be considered, such as neurological or cardiac events, and the tests should be followed up (NICE, 2015).

In the first instance making a differential diagnosis of UTI is helpful to suggest further testing. Making a differential diagnosis will need knowledge of normal and abnormal symptoms, history taking skills, investigations and practice. Additional symptoms of a UTI can include frequency; urgency; and/or strangury (the feeling of needing to pass urine despite having just done so); dysuria (pain or discomfort on passing urine); urine that is offensive smelling, cloudy, or contains blood; lower abdominal aches or pains; and aching sometimes accompanied with nausea, fatigue and sweats. (NICE 2015)

Older frail women (with or without catheters) may not have these features and can present with aching ribs, rigors, increased confusion, and a slightly elevated temperature, for example, 37.9 degrees centigrade or more than 1.5 degrees $\mathrm{C}$ above their normal temperature (NICE 2015)

\section{Using the urine dipstick test}

NICE (2014) state that adults with only slight or mild symptoms and no catheter should have a dipstick test performed on their urine to determine if leucocyte esterase and nitrites are present. With comprehensive and appropriate training most 
health care workers should be able to perform competent dipstick urine testing. Generally speaking, if urine testing does not show positive results for either of the above reagents then a diagnosis of UTI is unlikely. Urinary leucocytes indicate white blood cells in the urine and may give some helpful differentials (such as thrush or a vaginal discharge) and make a UTI moderately likely but if nitrites are also positive (with or without leucocytes) this makes a UTI very likely (NICE 2015).

In the older, female catheterised patient who is complaining of 'mild' symptoms e.g. offensive urine, slight pain or discomfort and fever - the HCW may consider a diagnosis of UTI. If an older female has severe symptoms (elevated temperature, more discomfort and possible first time or increasing confusion) the same method applies. Urine dipsticks are not that useful for diagnosis in this group as after catheterisation there will always be a degree of inflammation and therefore protein and/or bacteria in the urine (which is physiological and does not need treatment (unless symptoms are present) (NICE 2015).

\section{Treatment options for both genders}

If patients have moderate to severe symptoms (fever, pain, dysuria, offensive urine) treatment should be started as soon as possible. If feasible a sample should be taken and tested but in some cases (weekends and at night) this may not be an option. By following local prescribing guidelines the most appropriate antibiotic can be chosen to speed recovery and reduce complications. There will options for treatment without sample results (NICE 2015)

It is considered that due to anatomical differences males with a positive urine dipstick should have a further test on the urine - microbiology, culture and sensitivity with treatment of UTIs lasting seven days (so sending the sample to the GP who will send it for lab testing) Persistent or recurrent UTIs with or without other relevant long term conditions in either gender needs further investigation. It has been shown that while half all of the women in the UK will have a UTI at least once in their lifetime the incidence in males is only 1 in 2000 per year (NHS choices 2013)

UTIs in female patients are generally considered to be uncomplicated- so of short duration and mostly without longer term problems so these can usually be treated with a 3 day course of antibiotics according to local guidelines. A watch and wait option with increased fluids and analgesia is also feasible in those with mild symptoms. in males this is rarely the case - NICE (2015) has some excellent algorhitms and scenarios to explain the differences. NICE 2015

In older patients more care must be taken to prescribe according the patients drug allergies, other medications they may be taking and their current health. For instance, a poor kidney function (and /or low GFR) must be considered when prescribing many of the common antibiotics used for treating UTIs for example trimethoprim and nitrofurantoin. HCWs are in an ideal position to check that the guidelines for prescribing these are followed (RPS 2016) and need to keep up to date.

However one of the greatest challenges of testing urine in the elderly can be the collection of the sample. In the mobile, young patient a mid-stream urine specimen 
or clean catch specimen of urine is usually sufficient to test and/or send if indicated. In older people as with babies and children more innovative and inventive ways of catching the urine may be needed as they are unable to produce urine when requested (Lee and Arbuckle 2009).

There are many devices available A urine collection pot put into a commode to catch urine when the patient uses this (clean and unused), an insert into an incontinence pad (Tena $U$ Test)(but this must be used quickly ideally within 10 minutes to reduce erroneous findings), pads that collect urine that can be syringed up using either a syringe or a needle and syringe such as those offered at Redland healthcare (literally pushing the needle into the pad and withdrawing urine for the sample) and small pads containing reactor strips that can give immediate diagnostic criteria (Lee and Arbuckle 2009; Rao et al 2003) Samples collected in this way must be labelled and state the mode of collection on the microbiology form.

All of these collection methods are subject to price, availability, user bias and choice. But to improve diagnosis when urine is hard to collect they are invaluable and choices should be there for HCWs to make to improve diagnostic procedures. Once urine has been collected using a red top boric acid container if the sample needs to be kept overnight will reduce over growth of bacteria (SIGN 2013). This is preferable to a white top bottle which is useful only if the urine is going to be tested immediately.

\section{$\underline{\text { UTIs and catheters }}$}

Catheters predispose patients to infection (NICE 2015). Bacteria can be introduced through poor hygiene on insertion, manipulation of the catheter, incorrect placement of a drainage bag (too high allowing back flow) and a route of infection through which bacteria can pass. Bateruria (the presence of bacteria in the urine) is common in those with catheters and should not be used to diagnose infections. Several factors increase the likelihood of catheter-associated infections; these are (SIGN 2013):

Patients who are immunocompromised or debilitated

- Patients with diabetes or chronic kidney disease (CKD)

- Poor care of the catheter and drainage system

- Patients of the female gender

- Diarrhoea or faecal incontinence.

Current suggested criteria for diagnosing a UTI in catheterised patients is based around several algorithms (The SIGN algorithm (see figure 12013 )) is very useful as a baseline guide for suspected UTIs in catheterised residents in nursing homes and it suggests that the presence of one of the following symptoms should stimulate antibiotic therapy or at the very least a more comprehensive assessment.

- new tenderness around the ribs

- rigors

- new onset delirium 
- fever greater than $37.9 \mathrm{C}$ or $1.5 \mathrm{C}$ above baseline on two occasions during 12 hours

So in catheterised patients who present with fever:

- look for associated local (loin or supra-pubic tenderness) or systemic features

- exclude other potential sources of infection

- send off an appropriately taken urine sample for culture to determine the infecting organism and susceptibility to antibiotics ( via the GP)

- consider antibiotic therapy taking into account the severity of the presentation and any comorbidity factors after consulting with the managing physician or independent prescriber.

Only send urine samples for laboratory culture if the patient has clinical sepsis, not because the appearance or smell of the urine suggests that bacteriuria is present. Seek medical advice if unsure (NICE 2015.)

The following algorithm (figure 2) is useful from Public Health England but do compare and contrast it with the simpler table from SIGN 88 which specifically mentions UTIs in older people. Also listed are some of the guidelines available for reference

\section{Conclusion}

UTIs are a substantial problem in the elderly who may need frequent medical attendance and treatment-even hospitalisation. Promoting fluid intake may help to reduce dehydration and so reduce UTI risk. Dipstick testing and understanding the signs and symptoms of UTIs in general and how to overcome the hurdle of collection will improve the speed of diagnosis, patient outcomes and reduce morbidity and mortality. Training, national and local guidelines should be used in addition to national algorithms which are for guidance only. Below are some of the useful guidelines referred to in this article and which are easy to access on line.

\begin{tabular}{|l|l|}
\hline Guideline & Note \\
\hline $\begin{array}{l}\text { National Institute for Health } \\
\text { and Care Excellence (2015) }\end{array}$ & $\begin{array}{l}\text { Detailed guidelines and quality } \\
\text { standard on the management } \\
\text { of UTIs and use of scenarios to } \\
\text { illustrate the management }\end{array}$ \\
\hline $\begin{array}{l}\text { Scottish Intercollegiate } \\
\text { Guidelines Network (2013) }\end{array}$ & $\begin{array}{l}\text { Detailed guidelines on the } \\
\text { management of UTIs and use of } \\
\text { scenarios to illustrate the } \\
\text { management }\end{array}$ \\
\hline Local anti-microbial guidelines & $\begin{array}{l}\text { Used in complex or uncertain } \\
\text { cases }\end{array}$ \\
\hline
\end{tabular}

\section{References}


Alzheimer's Society (2011) Urinary tract infection (UTI) and dementia. http://bit. ly/1DijBwB (accessed $21^{\text {st }}$ August 2016)

Beveridge L, Davey P, Phillips G and McMurdo M (2011) Optimal management of urinary tract infections in older people. Clin Interv Aging. 2011; 6: 173-180.

Health Protection Agency and British Infection Association (2011) Diagnosis of UTI. Quick Reference Guide for Primary Care. bit.ly/1Esuemg (accessed $21^{\text {st }}$ August 2016)

Lally F and Crome P (2007) Understanding frailty. Postgrad Med J. 2007 Jan; 83(975): 16-20. doi: 10.1136/pgmj.2006.048587

Lee EJ and Arbuckle TE (2009) Urine-sampling methods for environmental chemicals in infants and young children. J Expo Sci Environ Epidemiol. 2009 Nov;19(7):625-33. doi: 10.1038/jes.2009.36. Epub 2009 Jun 24.

National Institute for Health and Care Excellence (2013) NICE guidelines [CG161]. Falls: assessment and prevention of falls in older people. http://www. nice.org.uk/guidance/cg161(accessed $21^{\text {st }}$ August 2016)

National Institute for Health and Care (2015) Urinary Tract infections in adults. https://www.nice.org.uk/guidance/qs90 (accessed 21st August 2016)

NHS Choices (2014) Urinary tract infections in adults - Prevention. bit. ly/1GkQIIS (accessed $21^{\text {st }}$ August 2016)

Rao S, Bhatt J, Houghton C and Macfarlane P (2009) An improved urine collection pad method: a randomised clinical trial. Arch Dis Child 2004;89:773-775 doi:10.1136/adc.2003.037770

Redland Healthcare (2016) Urine collection devices http://www.redlandhealthcare.co.uk/Catalogue/Continence-Care/UTI-Testing/UrineCollection-Kit accessed 1.9.16

Royal Pharmaceutical Society (2016) Prescribing competency framework. http://www.rpharms.com/unsecure-support-resources/prescribing-competencyframework.asp (accessed 22nd August)

Scottish Intercollegiate Guidelines Network (2013) SIGN Guideline 88: Management of suspected bacterial urinary tract infection in adults. http://www.sign.ac.uk/guidelines/fulltext/88 (accessed $21^{\text {st }}$ August 2016)

Tena U Test (2016) https://www.google.co.uk/search?q=tena+u+kit\&ie=utf-8\&oe=utf-

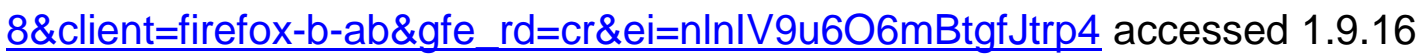




\section{Figure 1}

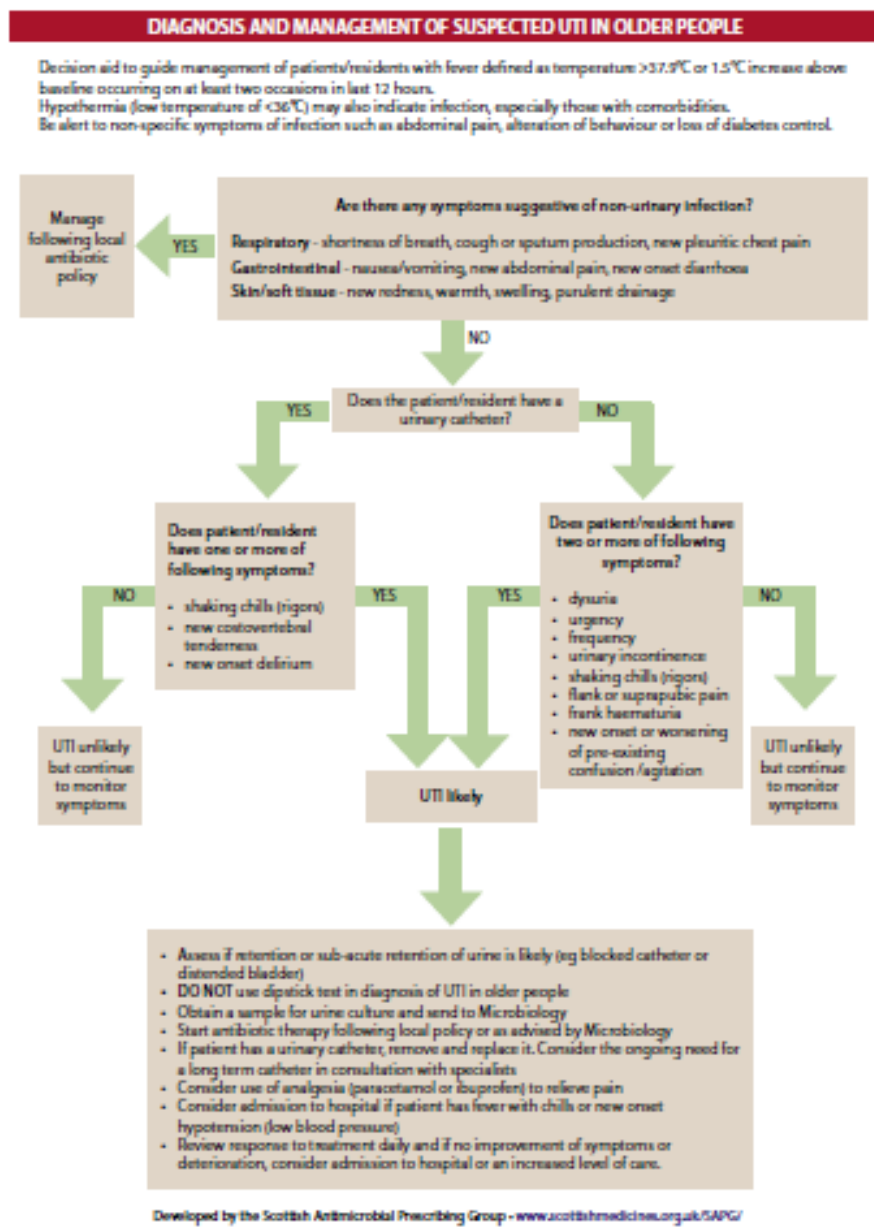


URINARY SYMPTOMS IN ADULT WOMEN $<65$ DO NOT CULTURE ROUTINELY ${ }^{1-5}$

In sexually active young men and women with urinary symptoms consider $\underline{\text { Chlamydia }}_{\text {trachomatis }^{\mathrm{C}}}$


\section{URINE CULTURE IN WOMEN AND MEN > 65 YEARS}

- Do not send urine for culture in asymptomatic elderly with positive dipsticks

- Only send urine for culture if two or more signs of infection, especially dysuria, fever $>38^{\circ}$ or new incontinence. ${ }^{4,5 C}$

- Do not treat asymptomatic bacteriuria in the elderly as it is very common. ${ }^{\mathrm{B}+}$

- Treating does not reduce mortality or prevent symptomatic episodes, but increases side effects \& antibiotic resistance. ${ }^{2,3, \mathrm{~B}+}$

\section{URINE CULTURE IN WOMEN AND MEN WITH CATHETERS}

- Do not treat asymptomatic bacteriuria in those with indwelling catheters, as bacteriuria is very common and antibiotics increase side effects and antibiotic resistance. ${ }^{\mathrm{B}+}$

- Treatment does not reduce mortality or prevent symptomatic episodes, but increase side effects \& antibiotic resistance. ${ }^{2,3, \mathrm{~B}+}$

- Only send urine for culture in catheterised ${ }^{7 \mathrm{~B}-}$ if features of systemic infection. ${ }^{1,5,6 \mathrm{C}}$ However, always:

Exclude other sources of infection. ${ }^{\mathrm{C}}$

Check that the catheter drains correctly and is not blocked.

Consider need for continued catheterisation.

If the catheter has been in place for more than 7 days, consider changing it before/when starting antibiotic treatment. ${ }^{1,6 \mathrm{C}, 8 \mathrm{~B}+}$

Do not give antibiotic prophylaxis for catheter changes unless history of symptomatic UTIs due to catheter change. ${ }^{9,10 \mathrm{~B}+}$ 


\section{WHEN ELSE SHOULD I SEND A URINE FOR CULTURE?}

- Pregnancy: If symptomatic, for investigation of possible UTI. ${ }^{1 \mathrm{~B}}$

In all at 1 st antenatal visit - as asymptomatic bacteriuria is associated with pyelonephritis \& premature delivery. ${ }^{1,2 \mathrm{~B}+}$

- Suspected pyelonephritis ${ }^{3 \mathrm{C}}$ (loin pain and fever).

- Suspected UTI in men. ${ }^{1,4 \mathrm{C}}$

- Failed antibiotic treatment or persistent symptoms.

E. coli with Extended-spectrum Beta-lactamase enzymes are increasing in the community.

ESBLs are multi-resistant but usually remain sensitive to nitrofurantoin or fosfomycin. ${ }^{7,8 \mathrm{~B}+, 9 \mathrm{~A}+}$

- Recurrent UTI, abnormalities of genitourinary tract, renal impairment - more likely to have a resistant strain. 